\title{
Análise plurianual da qualidade das águas de bacia tributária do Pantanal brasileiro
}

Os dados dos programas de monitoramento, têm sido pouco utilizados para subsidiar a tomada de decisões dos gestores das bacias hidrográficas. Diversas ferramentas podem ser adotadas, mas é fundamental que a primeira etapa seja através de análise descriminante. Esta análise, permite ao gestor distinguir, dentro de um conjunto de variáveis, quais são as que podem ser eleitas como indicadoras de processos de impacto (singularidades) daquelas que são provavelmente um atributo variável a longo prazo no sistema (intrínseco). O objetivo deste estudo é identificar e avaliar a estrutura intrínseca e as singularidades do corpo hídrico, com base nas variáveis físicas, químicas e biológicas de qualidade das águas, da Bacia do Rio Vermelho/MT, usando dados de monitoramento de longa duração (2006 a 2017), tendo como foco estabelecer um critério técnico para priorização de ações de conservação na bacia. A metodologia consiste no emprego de métodos estatísticos multivariados, da análise fatorial (AF) e da análise de componentes principais (ACP). Os resultados indicaram que os métodos estatísticos são eficientes para ranquear os parâmetros, de modo que os parâmetros: cor, demanda química de oxigênio, nitrogênio total, coliformes totais, cloreto, resíduo não filtrável, Escherichia coli, fosforo total, turbidez, resíduo total possuem maior peso na variância e são as variáveis características das alterações do corpo hídrico enquanto que: temperatura do ar, temperatura da água; nitrogênio amoniacal; nitrogênio nitrito, pH; oxigênio dissolvido, DBO, ortofosfato, vazão média; e nitrato, podem ser caracterizadas pela baixa variância como as variáveis intrínsecas do rio, que são de ordem natural do corpo hídrico. As potencialidades e limitações da metodologia e dos dados são discutidas. Considerando a complexidade de usos múltiplos da bacia, que incluem áreas de produção agrícola, unidades de conservação, PCHs, áreas urbanas e terras indígenas, frente ao cenário de tendência de perda da qualidade das águas, recomenda-se que a Bacia do Rio Vermelho seja incluída como prioridade nas estratégias de manejo e conservação.

Palavras-chave: Gestão ambiental; Estatística multivariada; Análise de componentes principais; Análise fatorial.

\section{Multiannual analysis of the quality of waters in the tax basin of the Brazilian Pantanal}

\begin{abstract}
The data from the monitoring programs, have been little used to support the decision making of the managers of the hydrographic basins. Several tools can be adopted, but it is essentia that the first step is through discriminating analysis. This analysis allows the manager to distinguish, within a set of variables, which are the ones that can be chosen as indicators of impact processes (singularities) of those that are likely to be a long-term variable attribute in the (intrinsic) system. The objective of this study is to identify and evaluate the intrinsic structure and singularities of the water body, based on the physical, chemical and biological variables of water quality, in the Rio Vermelho Basin/MT, using long-term monitoring data (2006 to 2006). singularities of the water body, based on the physical, chemical and biological variables of water quality, in the Rio Vermelho Basin/MT, using long-term monitoring data (2006 to 2006 ). 2017), focusing on establishing a technical criterion for prioritizing conservation actions in the basin. The methodology consists of the use of multivariate statistical methods, factor analysis
(PA) and principal component analysis (PCA). The results indicated that the statistical methods are efficient to rank the parameters, so that the parameters: color, chemical oxygen demand,
total nitrogen, total coliforms, chloride, non-filterable residue, Escherichia coli, total phosphorus, turbidity, total residue have greater weight in the variance and are the characteristic variables total nitrogen, total coliforms, chloride, non-filterable residue, Escherichia coli, total phosphorus, turbidity, total residue have greater weight in the variance and are the characteristic variables of changes in the water body while: air temperature, water temperature; ammoniacal nitrogen; nitrogen nitrite, $\mathrm{pH}$; dissolved oxygen, BOD, orthophosphate, medium flow; and nitrate, can be characterized by low variance as the intrinsic variables of the river, which are natural in the water body. The potential and limitations of the methodology and data are discussed. Considering the complexity of multiple uses of the basin, which include agricultural production areas, conservation units, SHP, urban areas and ind
\end{abstract}

Keywords: Environmental Management; Multivariate Statistics; Principal Component Analysis; Factor analysis.

Topic: Engenharia Ambiental

Reviewed anonymously in the process of blind peer.
Received: $10 / 02 / 2020$

Approved: 07/03/2020
Mayara Oliveira da Silva

Universidade Federal de Mato Grosso, Brasil

http://lattes.cnpq.br/2159069093530534

mayaraeaa@gmail.com

Ricardo Alves Olinda (ib

Universidade Estadual da Paraíba, Brasil

http://lattes.cnpq.br/7767223263366578

http://orcid.org/0000-0002-0509-8428

ricardo.estat@yahoo.com.br

\section{Camila Leonardo Mioto (ic}

Universidade Federal de Mato Grosso, Brasil

http://lattes.cnpq.br/2846355335199799

http://orcid.org/0000-0002-6951-9527

ea.mioto@gmail.com
Domingos Sávio Barbosa (iD

Universidade Federal de Mato Grosso, Brasi

http://lattes.cnpq.br/6896725721269796

http://orcid.org/0000-0001-6793-0956

domingosbar@gmail.com
Referencing this:

SILVA, M. O.; OLINDA, R. A.; MIOTO, C. L.; BARBOSA, D. S.. Análise plurianual da qualidade das águas de bacia tributária do Pantanal brasileiro. Revista Ibero Americana de Ciências Ambientais, v.11, n.2, p.172-181, 2020. DOI: http://doi.org/10.6008/CBPC21796858.2020 .002 .0019

DOI: 10.6008/CBPC2179-6858.2020.002.0019 


\section{INTRODUÇÃO}

Nas últimas décadas, diversos estudos têm se concentrado na concepção de redes de monitoramento e novas metodologias têm sido propostas, onde se destaca os estudos utilizando abordagens estatísticas multivariadas de dados, através da análise fatorial (AF) e análise de componentes principais (ACP) (MAVUKKANDY et al., 2014; GARDIMAN JUNIOR et al., 2018). Uma série de estudos, tem adotado o uso de métodos multivariados com a finalidade de selecionar variáveis visando otimizar protocolos de monitoramento, reduzindo o número de variáveis a serem avaliadas bem como as estações de amostragem (NONATO et al., 2007; MAS et al., 2010; ROCHA et al., 2014; ALVES et al., 2018). Contudo, a estratégia reducionista não soluciona por completo os objetivos do procedimento de monitoramento ambiental de longo prazo e podem incorrer em perda significativa de informações.

Em outra linha de análise, face as crescentes preocupações no Brasil acerca da resposta a desastres ambientais envolvendo o meio aquático, os dados históricos de monitoramento são cruciais para aferir tanto o dano quanto as metas de recuperação. A estrutura intrínseca de um conjunto de dados de qualidade das águas, descreve uma característica que é própria e essencial de um sistema lótico enquanto a singularidade descreve um aspecto diferencial, que pode estar relacionado com um impacto ambiental. Esta análise, permite ao gestor distinguir, dentro de um conjunto de variáveis, quais são as que podem ser eleitas como indicadoras de processos de impacto (singularidades) daquelas que são provavelmente um atributo pouco variável no sistema (intrínseco).

A análise multivariada permite ainda análise das correlações entre as variáveis avaliadas (p. ex. Teste de Correlação de Pearson), discriminando seu peso de importância com base na explicação da variância (p. ex. Análise de Componentes Principais), possibilitando inclusive a criação de grupos de variáveis (p. ex. Análise de Cluster) (CRUZ et al., 2018). Este conjunto de análises permite inferir quanto a estrutura intrínseca dos dados de uma dada região, ou seja, suas características-chave, gerando um conjunto de variáveis ranqueáveis por peso de importância, que podem ser utilizados como descritores classificatórios dos sistemas aquáticos. $\mathrm{O}$ objetivo deste estudo é identificar e avaliar estrutura intrínseca e as singularidades do corpo hídrico, com base nas variáveis físicas, químicas e biológicas de qualidade das águas, da Bacia do Rio Vermelho/MT, usando dados de monitoramento de longa duração (2006 a 2017).

\section{MATERIAIS E MÉTODOS}

\section{Caracterização da área de estudo}

A região hidrográfica do Alto Paraguai está localizada na porção Oeste do Brasil, compreendendo os territórios de Mato Grosso e Mato Grosso do Sul. No trecho nordeste da bacia do Alto Paraguai, encontra-se a sub-bacia do São Lourenço que é formada pelo rio São Lourenço e seus principais afluentes pela margem esquerda como os rios Pombas e Córrego Prata, e pela margem direita, os rios Vermelho e São Pedro (MMA, 2006). São alguns dos principais contribuintes do Pantanal brasileiro, sendo um dos sistemas aquáticos que promovem a conectividade entre os biomas Cerrado e Pantanal. 
A sub-bacia do rio Vermelho, está localizada na região sudeste do Estado de Mato Grosso situa-se em região do bioma Cerrado, e possui clima tropical úmido. A bacia ocupa uma área de cerca de 150.802 ha (SOUZA et al., 2014). O Rio Vermelho tem como afluente de águas pela margem direita as sub-bacias do Paraíso, Tombador, Areia, Tadarimana, Jurigue, Ponte de Pedra, e pela margem esquerda, as sub-bacias do Poxóreu, Grande, Bagaréu, Arareau Míau e São Lourenço.

O uso e ocupação da bacia do rio vermelho é destinado majoritariamente para atividades econômicas para exportação de commodities agrícolas, tais como a pecuária de corte e produção grãos (soja e milho), seguida por culturas temporárias. Há a presença de mata ciliar remanescente, além de áreas urbanizadas (SOUZA, 2015).

A área de maior concentração demográfica da bacia se restringe ao espaço ocupado pela mancha urbana da sede municipal de Rondonópolis. O município de Rondonópolis pelo último censo tinha população de 195.476 pessoas, com densidade demográfica de 47,00 hab/ $\mathrm{km}^{2}$ e população estimada em 2019 de 232.491 pessoas (IBGE, 2010). A área em estudo é apresentada na Erro! Fonte de referência não encontrada. .

Sob o ponto de vista estratégico do uso da água, a Bacia do Rio Vermelho apresenta uma condição de alta complexidade e necessita de atenção prioritária. Inclusas na área de drenagem da bacia, estão três terras indígenas (Tadarimana, Jarudore e Tereza Cristina), sendo a terra indígena Tadarimana com cerca de 9.785 ha, equivalente a área urbana atual do Município de Rondonópolis. Também estão localizadas duas grandes unidades de conservação estadual (Parque Estadual Dom Osório Stoffel, 6.421,69 ha e RPPN João Basso 3.624,57 ha). Compartilham ainda no território da bacia, duas PCHs (Ponte de Pedra, 176 MW e Rondonópolis 26,60 MW) (VICENTE, 2015).

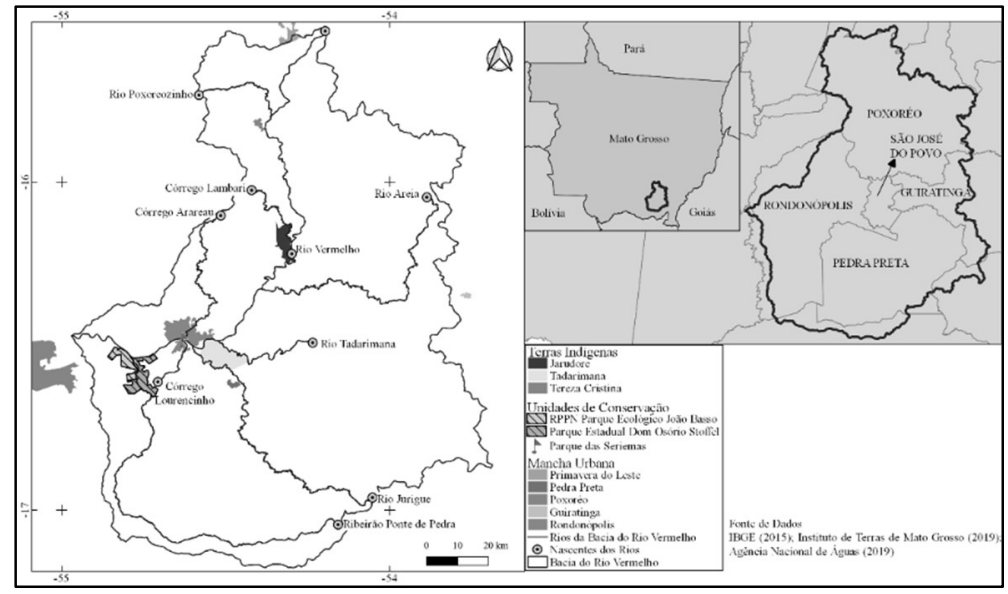

Figura 1: Mapa de localização da Bacia do Rio Vermelho.

\section{Dados de qualidade das águas}

Os dados do monitoramento da qualidade das águas da bacia do Rio Vermelho estão divulgados nos relatórios de monitoramento da qualidade das águas da região hidrográfica do Alto Paraguai, realizada pela coordenadoria de monitoramento de qualidade ambiental, operada pela SEMA-MT. Atualmente, as estações de coleta estão cadastradas no banco de dados da Agência Nacional das Águas ANA - HIDRO. Os dados de 
qualidade da água dessas estações podem ser consultados no site da ANA/HIDROWEB ${ }^{1}$, e as estações de amostragem estão detalhadas na Erro! Fonte de referência não encontrada.. Nesse estudo, utilizou-se um conjunto de dados de quatro estações de amostragem, no período de 2006 a 2017, de periodicidade trimestral.

Tabela 1: Caracterização das estações de coleta para monitoramento da qualidade das águas da Bacia do Rio Vermelho.

\begin{tabular}{|l|l|l|l|}
\hline Estação & Código Estação & Cidade & Coordenadas Geográficas \\
\hline Jarudore & VEM015 & Jarudore & $\begin{array}{l}16^{\circ} 11^{\prime} 46,73^{\prime \prime} \mathrm{S} \\
54^{\circ} 18^{\prime} 3,45^{\prime \prime} \mathrm{W}\end{array}$ \\
\hline Rondonópolis & & & $\begin{array}{l}16^{\circ} 28^{\prime} 46,49^{\prime \prime} \mathrm{S} \\
54^{\circ} 39^{\prime} 6,45^{\prime \prime} \mathrm{W}\end{array}$ \\
\hline Ponte de Pedra & VEM093 & Rondonópolis & $16^{\circ} 31^{\prime} 47,04^{\prime \prime} \mathrm{S}$ \\
& & Rondonópolis & $54^{\circ} 47^{\prime} 27,47^{\prime \prime} \mathrm{W}$ \\
\hline Pedra Preta & VEM111 & & $16^{\circ} 36^{\prime} 24,00^{\prime \prime} \mathrm{S}$ \\
& & Pedra Preta & $54^{\circ} 27^{\prime} 31,00^{\prime \prime} \mathrm{W}$ \\
\hline
\end{tabular}

\section{Análise estatística multivariada}

O banco de dados foi estruturado e analisado, sendo que nesta análise foi avaliada uma matriz de ordem $126 \times 26$ (linhas $\times$ colunas), tendo como variáveis os 26 parâmetros de qualidade de água e como observações os meses de amostragem nas estações descritas na Erro! Fonte de referência não encontrada.. As análises multivariadas, análise fatorial (AF) e da análise de componentes principais (ACP) foram realizadas no software R (R CORE TEAM, 2019).

Para a AF o método de extração utilizado foi via ACP e rotação Varimax foi utilizada, obtendo-se pesos altos para cada variável em um único fator e pesos baixos ou moderados nos demais fatores. Na ACP dos dados de monitoramento de qualidade de água da bacia do Rio Vermelho, os componentes principais foram extraídos da matriz de correlação, eliminando possíveis influências em virtude das diferentes ordens de magnitude dos parâmetros de qualidade de água. Para estimação do número de componentes principais utilizou-se o critério de Kaiser (KAISER, 1985), denominado neste estudo de fator k. Sob este critério, foram analisados os componentes com autovalores maiores que 1,0.

O Critério de escolha do melhor ajuste fatorial seguiu os seguintes parâmetros: Raíz Média Quadrada dos Resíduos (RMSR), esse valor deve estar próximo de 0; Raiz Quadrada do Erro Médio de Aproximação (RMSEA) deve estar abaixo de 0,05, Índice Tucker-Lewis (TLI) considerando-se aceitável acima de 0,9 (REVELLE, 2017).

\section{RESULTADOS E DISCUSSÃO}

Na Tabela 2 apresenta-se o resultado dos autovalores, bem como a porcentagem de variância explicada por cada fator, e a variância acumulada pelas mesmas. A classificação dos fatores foi feita com base nos fatores cujos valores próprios são superiores a 1,0 sendo este critério é sugerido por Critério de Kaiser (1958): o número de fatores retidos deve ser igual ao número de autovalores maiores que 1,0. A ideia básica

\footnotetext{
${ }^{1}$ http://www.snirh.gov.br/hidroweb/publico/apresentacao.jsf
} 
do critério é manter no sistema novas dimensões que representem pelo menos a informação de variância de uma variável original.

Dos resultados da AF aplicada ao conjunto de dados, oito fatores foram considerados mais representativos, pois representam $67.64 \%$ da variância total, sobre os parâmetros de qualidade da água, onde o Fator 1 (F1) foi responsável por cerca de 18.78\%, Fator 2 (F2) por 10.87\%, Fator 3 (F3) por 8.28\%, de explicação das variações dos dados.

Tabela 2: Autovalores, porcentagem da variância explicada e proporção acumulada pelos componentes dos parâmetros de qualidade das águas da Bacia do Rio Vermelho.

\begin{tabular}{|l|l|l|l|}
\hline Fator & Autovalor & \% Variância & \% Acumulada da Variância \\
\hline 1 & 4,51 & 18,78 & 18,78 \\
\hline 2 & 2,61 & 10,87 & 29,65 \\
\hline 3 & 1,99 & 8,28 & 37,92 \\
\hline 4 & 1,78 & 7,41 & 45,33 \\
\hline 5 & 1,60 & 6,65 & 51,98 \\
\hline 6 & 1,35 & 5,61 & 57,59 \\
\hline 7 & 1,25 & 5,23 & 62,82 \\
\hline 8 & 1,16 & 4,82 & 67,64 \\
\hline 9 & 0,99 & 4,12 & 71,76 \\
\hline 10 & 0,90 & 3,74 & 75,50 \\
\hline 11 & 0,85 & 3,53 & 79,02 \\
\hline 12 & 0,74 & 3,07 & 82,09 \\
\hline 13 & 0,67 & 2,78 & 84,88 \\
\hline 14 & 0,61 & 2,55 & 87,43 \\
\hline 15 & 0,55 & 2,30 & 89,73 \\
\hline 16 & 0,51 & 2,11 & 91,85 \\
\hline 17 & 0,44 & 1,84 & 93,69 \\
\hline 18 & 0,35 & 1,45 & 95,14 \\
\hline 19 & 0,32 & 1,34 & 96,48 \\
\hline 20 & 0,31 & 1,30 & 97,79 \\
\hline 21 & 0,18 & 0,75 & 98,54 \\
\hline 22 & 0,14 & 0,57 & 99,11 \\
\hline 23 & 0,12 & 0,52 & 99,63 \\
\hline 24 & 0,09 & 0,37 & 100,00 \\
\hline & & \\
\hline
\end{tabular}

O modelo fatorial utilizado foi gerando os fatores por meio da máxima verossimilhança, e os valores dos pesos das variáveis nos fatores podem ser observados na Erro! Fonte de referência não encontrada.. Quando ocorre a rotação Varimax, diminui-se a contribuição das variáveis com menor significância e aumenta-se a contribuição das que possuem maior significância (DAMÁsIO, 2012). A diferença entre componentes principais e fatores é que enquanto as componentes principais são combinações lineares de variáveis de qualidade de água observáveis, os fatores podem incluir variáveis não-observáveis, hipotéticas e "latentes" (NEISSE et al., 2016).

A Tabela 3 mostra a matriz fatorial obtida como resultado da AF, os valores em negrito apresentam as maiores correlações entre os parâmetros e cada um dos Fatores e os valores sublinhados destacam as cargas maiores que 0,4 .

Na Tabela 3 estão representados os pesos das variáveis nos fatores, sendo que no F1 estão: Cloreto, resíduo não filtrável, turbidez, resíduo total; F2: Cor, demanda química de oxigênio, nitrogênio total, coliformes totais coliformes totais, fosforo total. O F1 tende a ser um fator geral com quase todas variáveis com carga significante e explica a quantia maior da variância. O segundo Fator e os seguintes são então 
baseados na quantia residual de variância. Cada fator explica porções excessivamente menores de variância (HAIR JUNIOR et al., 2009). Neste caso o Fator 1 e 2 podem ser classificados como singulares faces a alta importância para a variância e podem ser associados a perda de qualidade das águas.

Tabela 3: Matriz dos pesos das variáveis nos fatores e comunidades dos parâmetros de qualidade da água da Bacia do Rio Vermelho.

\begin{tabular}{|l|l|l|l|l|l|l|l|l|l|}
\hline & F1 & F2 & F3 & F4 & F5 & F6 & F7 & F8 & Com \\
\hline VMD & 0,21 & 0,20 & $-0,11$ & 0,11 & 0,20 & $-0,05$ & $-0,21$ & $-0,16$ & 6,0 \\
\hline COR & 0,12 & $\mathbf{0 , 5 7}$ & 0,05 & $-0,02$ & 0,06 & 0,01 & $-0,03$ & 0,01 & 1,1 \\
\hline COND & $-0,04$ & 0,10 & $\mathbf{0 , 4 9}$ & $-0,20$ & 0,02 & $-0,22$ & 0,24 & 0,22 & 2,9 \\
\hline DQO & 0,04 & $\mathbf{0 , 7 6}$ & 0,15 & 0,03 & $-0,04$ & 0,05 & $-0,08$ & $-0,19$ & 1,3 \\
\hline N-A & $-0,11$ & 0,00 & 0,04 & $-0,04$ & 0,29 & $-0,05$ & 0,07 & 0,00 & 1,5 \\
\hline N-NI & $-0,05$ & 0,01 & 0,02 & $-0,09$ & 0,15 & 0,23 & 0,07 & $-0,14$ & 3,2 \\
\hline N-TO & 0,30 & $\mathbf{0 , 5 1}$ & $-0,12$ & 0,04 & 0,19 & $-0,08$ & 0,07 & 0,17 & 2,5 \\
\hline CTO & 0,16 & $\mathbf{0 , 4 6}$ & 0,04 & 0,10 & 0,44 & $-0,19$ & 0,16 & $-0,10$ & 3,1 \\
\hline ALC & $-0,06$ & $-0,17$ & $\mathbf{0 , 7 8}$ & $-0,03$ & 0,19 & 0,01 & 0,09 & $-0,26$ & 1,5 \\
\hline ORT & $-0,04$ & 0,09 & $-0,05$ & 0,05 & 0,04 & 0,02 & 0,05 & $-0,23$ & 1,7 \\
\hline DTO & 0,09 & 0,01 & $\mathbf{0 , 8 3}$ & 0,07 & $-0,13$ & 0,41 & $-0,24$ & 0,23 & 1,9 \\
\hline CLO & $\mathbf{0 , 4 3}$ & 0,08 & 0,11 & 0,08 & 0,08 & $-0,08$ & 0,17 & 0,42 & 2,8 \\
\hline SULF & 0,10 & 0,31 & $\mathbf{0 , 6 8}$ & 0,10 & $-0,07$ & $-0,06$ & 0,12 & 0,45 & 2,4 \\
\hline RNF & $\mathbf{0 , 9 2}$ & 0,24 & 0,05 & 0,00 & $-0,06$ & $-0,04$ & 0,01 & 0,02 & 1,2 \\
\hline TAR & 0,01 & $-0,12$ & $-0,09$ & $\mathbf{0 , 9 8}$ & $-0,04$ & 0,02 & 0,03 & $-0,08$ & 1,1 \\
\hline TH2O & $-0,01$ & 0,14 & 0,04 & $\mathbf{0 , 7 7}$ & 0,12 & $-0,19$ & $-0,09$ & 0,03 & 1,3 \\
\hline OD & $-0,07$ & $-0,22$ & 0,04 & $-0,09$ & $-0,15$ & $\mathbf{0 , 6 4}$ & 0,17 & 0,03 & 1,6 \\
\hline E CO & 0,29 & 0,19 & $-0,05$ & 0,24 & $\mathbf{0 , 8 7}$ & 0,18 & $-0,12$ & $-0,05$ & 1,7 \\
\hline pH & 0,06 & $-0,01$ & 0,06 & $-0,02$ & 0,11 & 0,21 & $\mathbf{0 , 7 3}$ & $-0,06$ & 1,3 \\
\hline DBO & 0,17 & 0,16 & 0,03 & 0,24 & 0,05 & 0,25 & 0,03 & $-0,08$ & 3,9 \\
\hline N-NA & $-0,04$ & $-0,23$ & $-0,07$ & $-0,04$ & $-0,10$ & 0,13 & 0,03 & $-0,06$ & 2,7 \\
\hline FTO & 0,18 & $\mathbf{0 , 4 6}$ & $-0,16$ & $-0,02$ & $-0,04$ & $-0,06$ & 0,03 & $-0,10$ & 1,7 \\
\hline TURB & $\mathbf{0 , 9 3}$ & 0,11 & $-0,04$ & $-0,05$ & 0,03 & 0,00 & 0,03 & 0,07 & 1,1 \\
\hline RTO & $\mathbf{0 , 7 3}$ & 0,49 & 0,02 & 0,12 & 0,01 & 0,00 & $-0,11$ & 0,16 & 2,0 \\
\hline
\end{tabular}

Legenda: VMD: Vazão Média, COR: Cor, COND: Condutividade, DQO: Demanda química de Oxigênio, N-A: Nitrogênio Amoniacal, N-NI: N-NA: Nitrogênio Nitrato; N-TO: Nitrogênio Total, CTO: Coliformes Totais, ALC: Alcalidade, ORT: Ortofosfato, DTO: Dureza Total, CLO: Cloreto, SULF: Sulfato, RNF: Resíduo Não Filtrável, TAR: Temperatura do Ar, TH2O: Temperatura da Água, OD: Oxigênio Dissolvido, E CO: Escherichia coli, pH: pH, DBO: Demanda Bioquímica de Oxigênio, Nitrogênio Nitrito, FTO: Fósforo Total, TURB: Turbidez, RTO: Resíduo Total.

No F3 que explicam $8.28 \%$ da variância total, as principais variáveis foram: condutividade, alcalinidade, sulfeto, dureza total; podendo caracterizar com substâncias alcalinizantes, ou seja, ricas em sais de cálcio e magnésio que podem conter ainda sulfatos. No F4: a temperatura do ar e da água podendo ser denominado de fator Sazonal da Qualidade das Águas. Os demais componentes, basicamente reforçam a importância das variáveis descritoras da contaminação por efluentes e fontes de difusas associadas a nutrientes.

Seis parâmetros obtiveram peso menor ou igual a 0,30, são elas: vazão média, demanda bioquímica de oxigênio, nitrito, nitrato, nitrogênio amoniacal, ortofosfato. Julga-se que cargas fatoriais maiores que \pm 0,30 atingem o mínimo de significância; cargas de $\pm 0,40$ são consideradas mais importantes; e se as cargas são de $\pm 0,50$ ou maiores, elas são consideradas com significância prática (HAIR JUNIOR et al., 2009). Logo, esses parâmetros não obtiveram o valor do mínimo de significância em suas cargas fatoriais.

Com a técnica da análise fatorial foi possível obter resultados satisfatórios, pois a variabilidade (correlação) das variáveis está contida nas respectivas cargas fatoriais. Quanto maior a comunalidade, maior será o poder de explicação daquela variável pelo fator. Também é interessante destacar que os principais fatores de pressão sobre a qualidade das águas da Bacia do Rio Vermelho, identificados nos Relatório Anuais 
dos últimos 10 anos (SEMA, 2018) foram lançamento de efluentes e/ou poluição de origem difusa, resultado que coincide com os fatores encontrados pela AF.

A Erro! Fonte de referência não encontrada. apresenta o biplot ou seja a representação gráfica dos parâmetros de qualidade da água e as estações de amostragem, espacialmente, de forma que a espacialização das relações pode ficar mais clara, sendo que quanto menor o ângulo entre os vetores, mais correlacionadas estão as variáveis. Cada ponto no gráfico representa um par de correlação, ou seja, cada variável principal correlacionada com os componentes principais, indicando assim qual delas estão mais associadas aos componentes (LATTIN et al., 2011). É possível observar que a estação ação de Pedra Preta se diferencia das demais, demonstrando uma singularidade desta estação, sendo mais caracterizada pelas variáveis: sulfato, dureza total, alcalinidade e condutividade, parâmetros que foram característicos do F3.

As principais fontes de dureza são a dissolução de minerais contendo cálcio e magnésio, exemplificando, as rochas calcárias e os despejos industriais (VON SPERLING, 2007). Efluentes comumente apresentam alcalinidades altas devido à presença de silicatos e fosfatos. A alcalinidade alta em águas naturais pode inviabilizar seu uso para irrigação e pode ser indício de contaminação por efluentes industriais.

Segundo Boesch (2002) e Esteves (2011), a condutividade elétrica é um parâmetro que pode mostrar alterações na composição dos corpos d'água. Sendo um parâmetro importante para controlar e determinar o estado e a qualidade de água (PIÑEIRO DI BLASI et al., 2013). O uso do solo pode modificar diretamente a composição de uma água refletindo-se na condutividade elétrica (ESTEVES, 2011; VON SPERLING, 2005).

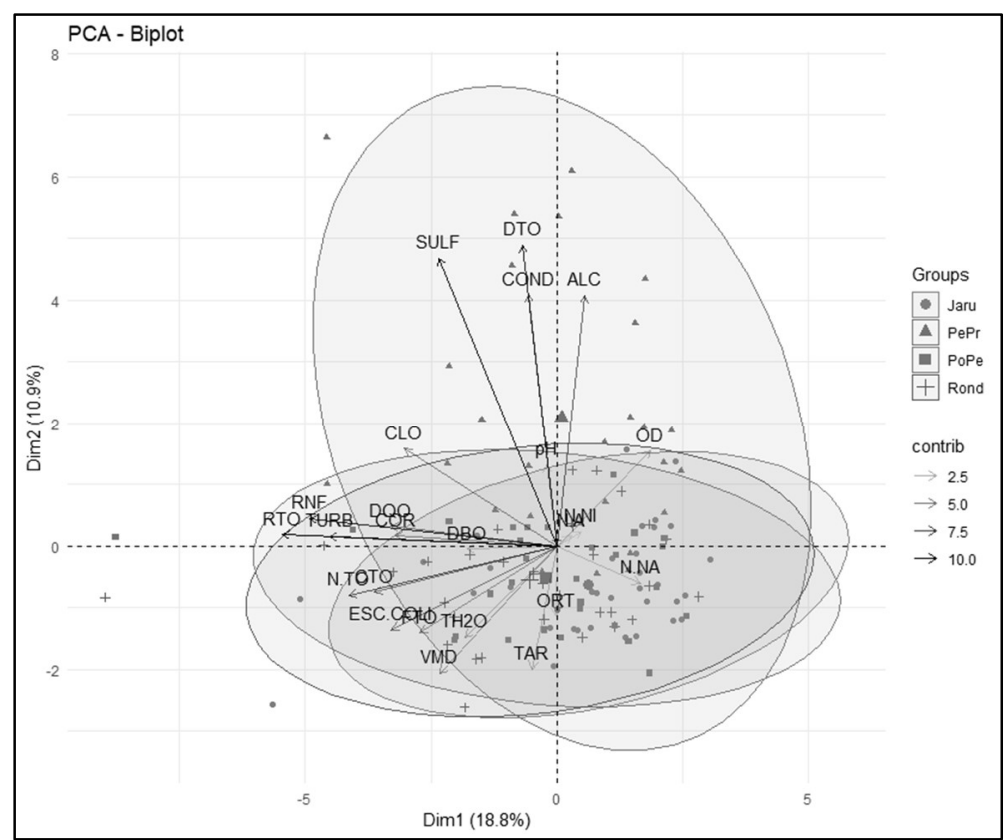

Figura 2: Biplot das variáveis de qualidade das águas da Bacia do Rio Vermelho em relação as estações de amostragem pela Análise de Componentes Principais (ACP). Legenda: VMD: Vazão Média, COR: Cor, COND: Condutividade, DQO: Demanda química de Oxigênio, N-A: Nitrogênio Amoniacal, N-NI: N-NA: Nitrogênio Nitrato; N-TO: Nitrogênio Total,

CTO: Coliformes Totais, ALC: Alcalidade, ORT: Ortofosfato, DTO: Dureza Total, CLO: Cloreto, SULF: Sulfato, RNF: Resíduo Não Filtrável, TAR: Temperatura do Ar, TH2O: Temperatura da Água, OD: Oxigênio Dissolvido, E.CO: Escherichia coli, pH: pH, DBO: Demanda Bioquímica de Oxigênio, Nitrogênio Nitrito, FTO: Fósforo Total, TURB: Turbidez, RTO: Resíduo Total, Jaru: Estação Jarudore, Pepr: Estação Pedra Preta, PoPe: Estação Ponte de Pedra, Rond: Estação Rondonópolis. 
$\mathrm{Na}$ análise de ACP representada pelo biplot da Erro! Fonte de referência não encontrada. verifica-se uma boa separação entre os períodos de chuva e estiagem, ou seja uma dissimilaridade entre os grupos. Podendo discriminar as variáveis que mais caracterizam o período de estiagem, sendo elas: oxigênio dissolvido, nitrogênio nitrato, ortofosfato, temperatura do ar, pH, nitrogênio nitrito.

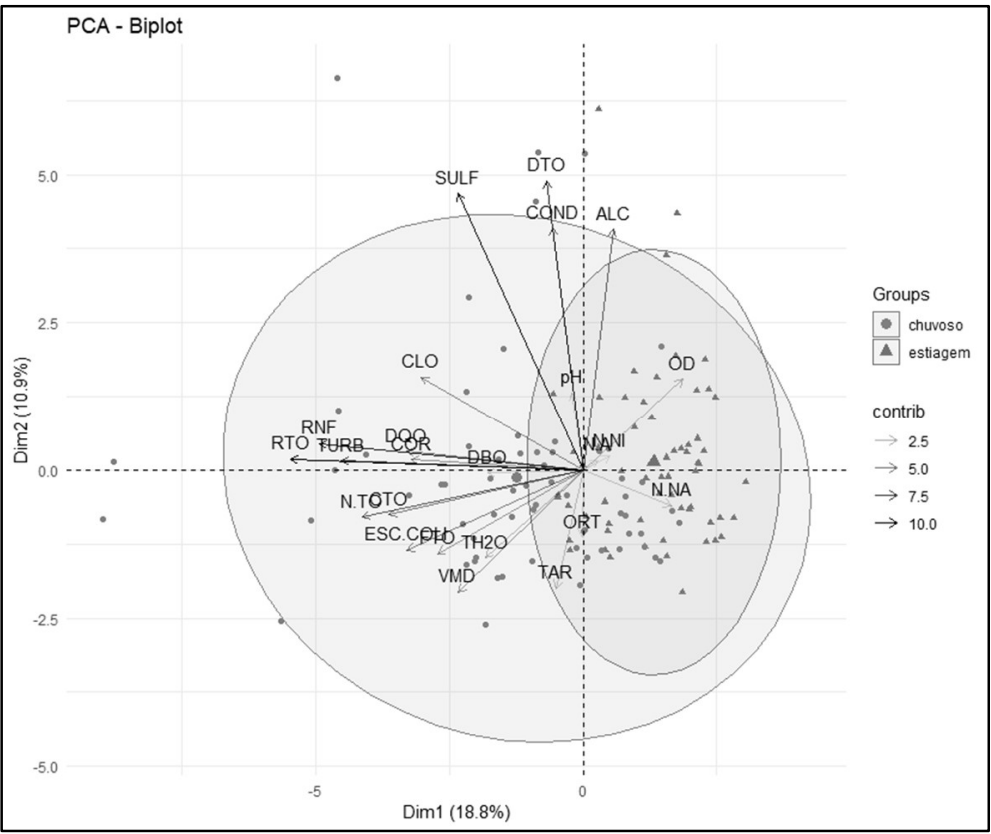

Figura 2: Biplot das variáveis de qualidade das águas da Bácia do Rio Vermelho em relação aos períodos chuvoso e estiagem pela Análise de Componentes Principais (ACP). Legenda: VMD: Vazão Média, COR: Cor, COND:

Condutividade, DQO: Demanda química de Oxigênio, N-A: Nitrogênio Amoniacal, N-NI: N-NA: Nitrogênio Nitrato; NTO: Nitrogênio Total, CTO: Coliformes Totais, ALC: Alcalidade, ORT: Ortofosfato, DTO: Dureza Total, CLO: Cloreto,

SULF: Sulfato, RNF: Resíduo Não Filtrável, TAR: Temperatura do Ar, TH2O: Temperatura da Água, OD: Oxigênio Dissolvido, E.CO: Escherichia coli, pH: pH, DBO: Demanda Bioquímica de Oxigênio, Nitrogênio Nitrito, FTO: Fósforo Total, TURB: Turbidez, RTO: Resíduo Total.

O biplot da Figura 2 demonstra que a chuva, entrada de material alóctone no corpo hídrico tem grande influência no comportamento do mesmo em relação as variáveis analisadas, levando à inferir de que a amostragem nos dois períodos (chuvoso e estiagem) é fundamental, pois os corpos de água são diferentes nestes períodos, com deterioração dos corpos de água nas chuvas com a entrada de material alóctone.

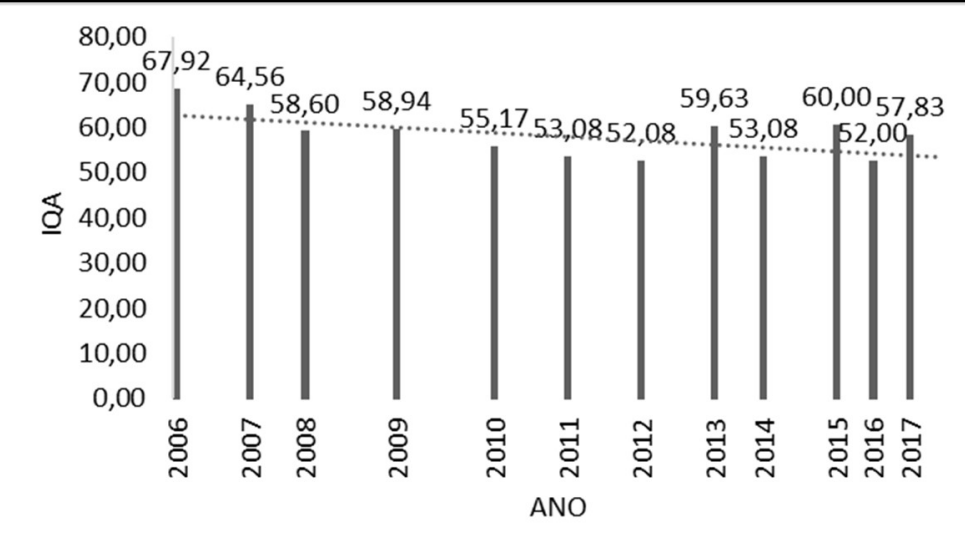

Figura 3: Gráfico de evolução do Índice de Qualidade das Águas (IQA) médio entre os anos de 2006 a 2017 da Bacia do Rio Vermelho. 
A Erro! Fonte de referência não encontrada. apresenta a variação do Índice de Qualidade da Água (IQA) médio entre os anos de 2006 a 2017, nota-se que em 2006 o valor médio era de 67,91 e ao fim do período de análise do dado estudo ele caiu em 10 sendo de 57,83, ambos os valores classificados como regular/médio, é possível também perceber os valores dos anos 2016 (52) e 2012 (52,08) tais valores próximos da classificação ruim. A análise da variação do IQA nos permite inferir uma tendência de queda na qualidade das águas da Bacia do Rio Vermelhos ao longo dos dez anos observados (2006 a 2017).

\section{CONCLUSÕES}

A AF promoveu a reorganização das variáveis de qualidade da água, de forma a caracterizar a estrutura intrínseca das variáveis descritoras da qualidade das águas da Bacia do Rio Vermelho. Tendo que as variáveis com maior variância foram as do F1 e F2, pode-se inferir que estas refletem as alterações que têm antropogênicas no corpo hídrico, e as com menor variância como sendo as variáveis intrínsecas do rio, ou seja que podem refletir as características naturais do sistema ou que exigirão alterações de longo prazo para serem afetadas.

No aspecto espacial, a estação de amostragem que demonstrou maior não similaridade do corpo hídrico com as demais foi a estação da Pedra Preta, sendo caracterizada principalmente por quatro variáveis: sulfato, dureza total, alcalinidade e condutividade. No aspecto temporal, foi possível descriminar as variáveis mais características da estação de estiagem, sendo elas: oxigênio dissolvido, nitrogênio nitrato, ortofosfato, temperatura do ar, $\mathrm{pH}$, nitrogênio nitrito. Apesar de muitos estudos indicarem a possibilidade de redução no número de variáveis nos programas de monitoramento, com base nos critérios de análise deste estudo, não foi observar a possiblidade de reduzir os escopos analíticos e sim, a discussão quanto a possibilidade de inclusão de novas variáveis.

\section{REFERÊNCIAS}

ALVES, J. P. H.; FONSECA, L. C.; CHIELLE, R. S. A.; MACEDO, L. C. B. Monitoring water quality of the Sergipe River basin: an evaluation using multivariate data analysis. RBRH, Porto Alegre, v.23, n.27, 2018. DOI: http://dx.doi.org/10.1590/2318-0331.231820170124

SEMA. Secretaria de Estado do Meio Ambiente. Monitoramento da Qualidade da Água do Estado de Mato Grosso. Cuiabá: SEMA/MT, 2018

BOESCH, D. F.. Challenges and opportunities for science in reducing nutrient over-enrichment of coastal ecosystems. Estuaries, v.25, n.4b, p.886-900, 2002. DOI: http://dx.doi.org/10.1007/BF02804914

CRUZ, D. G.; NOGUEIRA, F. C.; ROSSI, D. Q.; PEREIRA, T. M.; FERREIRA, R. F.. Análise fatorial multivariada aplicada na caracterização de contaminantes de um depósito de minério de ferro. Tecnol. Metal. Mater. Miner., São Paulo, v.15, n.3, p.248-253, 2018. DOI: http://dx.doi.org/10.4322/21761523.1432

DAMÁSIO, B. F.. Uso da análise fatorial exploratória em psicologia. Avaliação Psicológica, v.11, n.2, p.213-228, 2012.
ESTEVES, F.. Fundamentos de limnologia. 3 ed. Rio de Janeiro: Interciência, 2011.

HAIR JUNIOR, J. F.; WILLIAM, B.; BABIN, B.; ANDERSON, R. E.. Análise multivariada de dados. 6 ed. Porto Alegre: Bookman, 2009.

GARDIMAN JUNIOR, B. S.; GARCIA, G. O.; CECÍLIO, R. V.; 3 LOOS, R. A.; MAFIA, R. G.; ABAD, J. I. M.; SIMÃO, J. B. P.; BARBOSA, V. H. B.. Variáveis-chave para determinação da qualidade da água em microbacias com plantios de eucalipto. Ciência Florestal, Santa Maria, v.28, n.1, p.127140, 2018. DOI: http://dx.doi.org/10.5902/1980509831590

IBGE. Instituto Brasileiro de Geografia e Estatística. Censo 2010. Rio de Janeiro: IBGE, 2010.

KAISER, H. F.. The varimax criterion for analytic rotation in factor analysis. Psychometrika, v.23, n.3, p.187-200, 1958.

LATTIN, J.; CARROLL, J. D.; GREEN, P. E.. Análise de dados multivariados. São Paulo: Cengage Learning, 2011. 
MAS, S.; JUAN, A.; TAULER, R.; OLIVIERI, A. C.; ESCANDAR, G. $M$.. Aplication of chemometric methods to environmental analysis of organic pollutants: A review. Talanta, v.80, p.1052-1067, 2010. DOI:

https://doi.org/10.1016/j.talanta.2009.09.044

MAVUKKANDY, M. O.; KARMAKAR, S.; HARIKUMAR, P. S.. Assessment and rationalization of water quality monitoring network: a multivariate statistical approach to the Kabbini River (India). Environmental Science and Pollution Research International, v.21, n.17, p.10045-10066, 2014. DOI: http://dx.doi.org/10.1007/s11356-014-3000-y. PMid:24865500

MMA. Ministério do Meio Ambiente. Caderno da Região Hidrográfica do Paraguai. Brasília: MMA, 2006.

NEISSE, A. C.; HONGYU, K.. Aplicação de componentes principais e análise fatorial a dados criminais de 26 estados dos EUA. Engineering and Science, v.2, n.5, 2016. DOI: http://doi.org/10.18607/ES20165064

NONATO, E. A.; VIOLA, Z. G. G.; ALMEIDA, K. C. B.; SCHOR, H. H. R.. Tratamento estatístico dos parâmetros da qualidade das águas da bacia do alto curso do Rio das Velhas. Quím. Nova, v.30, n.4, p.797-804, 2007. DOI:

http://dx.doi.org/10.1590/S0100-40422007000400008

PIÑEIRO DI BLASI, J. I.; MARTÍNEZ TORRES, J.; GARCÍA NIETO P. J.; ALONSO FERNÁNDEZ, J. R.; DÍAZ MUÑIZ, C.; TABOADA, J.. Analysis and detection of outliers in water quality parameters from 'different automated monitoring stations in the Miño river basin (NW Spain). Ecological Engineering, v.60, p.60-66, 2013. DOI:

http://dx.doi.org/10.1016/j.ecoleng.2013.07.054
R CORE TEAM. R: A language and environment for statistical computing. R Foundation for Statistical Computing. Vienna, 2019.

REVELLE, W.. Psych: Procedures for Personality and Psychological Research, Northwestern University, Evanston, Illinois. 2017.

ROCHA, C. H. B.; FREITAS, F. A.; SILVA, T. M.. Alterações em variáveis limnológicas de manancial de Juiz de Fora devido ao uso da terra. R. Bras. Eng. Agríc. Ambiental, Campina Grande, v.18, n.4, p.431-436, 2014. DOI: http://dx.doi.org/10.1590/S1415-43662014000400011

SOUZA, A. V. V.. Influência dos usos e ocupação do solo na qualidade da água do rio Vermelho no sudeste de Mato Grosso. Dissertação (Mestrado em Geografia) Universidade Federal de Mato Grosso, Rondonópolis, 2015.

SOUZA, A. V. V.; LOVERDE-OLIVEIRA, S. M.. Análise da qualidade da água do Rio Vermelho em Mato Grosso: no período de cheia no ano de 2014. Biodiversidade, v.13, n.2, p.115-126, 2014.

VICENTE, T. C.. Gestão integrada de áreas protegidas: o potencial de Implantação do corredor ecológico cerrado/pantanal na bacia do Rio Vermelho/São Lourenço MT. Dissertação (Mestrado em Geografia) - Universidade Federal de Mato Grosso, Rondonópolis, 2015.

VON SPERLING, M.. Estudos de modelagem da qualidade da água de rios. Belo Horizonte: UFMG, 2007.

VON SPERLING, M.. Introdução à qualidade das águas e ao tratamento de esgoto. 3 ed. Belo Horizonte: UFMG, 2005.

A CBPC - Companhia Brasileira de Produção Científica (CNPJ: 11.221.422/0001-03) detém os direitos materiais desta publicação. Os direitos referem-se à publicação do trabalho em qualquer parte do mundo, incluindo os direitos às renovações, expansões e disseminações da contribuição, bem como outros direitos subsidiários. Todos os trabalhos publicados eletronicamente poderão posteriormente ser publicados em coletâneas impressas sob coordenação da Sustenere Publishing, da Companhia Brasileira de Produção Científica e seus parceiros autorizados. Os (as) autores (as) preservam os direitos autorais, mas não têm permissão para a publicação da contribuição em outro meio, impresso ou digital, em português ou em tradução. 